cells and $\mathrm{K}$ cells, with most of the reports emphasizing their similar though not identical properties. The next 4 sections cover the modulation of NK activity by a variety of mechanisms, including genetic factors, in vitro culture, interferon and inhibitory effects. The large section on specificity deals with receptors on NK cells and target-cell antigens, but despite a considerable amount of work in this area, there is little information on the nature of these structures.

The last two sections on NK cells cover the role of these cells in the host response to tumours. It is clear from these results that there is still little evidence that cells with NK activity have any effect on the growth of human tumours. NK activity is not generally present in the lymphocyte populations infiltrating human tumours or the cells in the draining lymph nodes, but the possibility that NK cells may inhibit tumour growth during the early stages requires further investigation. The final part of the book contains brief sections on natural immunity against microbial agents, lymphokine production and natural cytotoxicity by macrophages and granulocytes.

This book provides a comprehensive coverage of current results on NCMC, with contributions from most of the major laboratories working in this area. It consists of a collection of research papers and is therefore not intended as a general review of the subject, but it does form a very useful reference volume for those working on NCMC or related topics.

\section{R. Potter}

\section{Pathology of Tumours in Laboratory} Animals. Vol. II Tumours of the Mouse. V. S. Turusov (1979). Lyon: IARC (obtainable through H.M.S.O.). 669 pp. Sw. Fr. 100 net.

This volume is the second in a series, the first having dealt with tumours of the rat. The declared aim is to assist international efforts, particularly in carcinogenesis studies, by illustrating the range of tumours to be found in a given site and by suggesting a standard terminology for histological type. It is also regarded as a training manual for new workers.

Twenty-five chapters range comprehensively from the clinically important sites to the Harderian gland. The authors of each chapter have been required to include discussion of normal structure in each site, the biology of its tumours, their induction, and also the incidence and nature of "spontaneous" neoplasms. The quality of the more than 800 photomicrographs is mostly good. The legends have now been placed with their Figures, a major improvement on the first volume. Some chapters lack illustration of normal tissue, which is to be regretted in a "training manual".

It is probable that this volume, like the first, will find wide distribution among oncologists of all disciplines. The editor/ authors might therefore consider for a future edition, indicating whether and where particular tumours (and especially differentiated ones) remain available to other workers, by their maintenance as a line or in nitrogen storage.

\section{J. V. MOORE}

\section{N-nitroso Compounds Analysis, Forma- tion and Occurrence. Eds E. A. WALTER,} L. Griciute, M. Castegnaro and M. Borzsonyi (1981). Geneva: WHO. 841 pp. Sw. Fr. 70 net.

A large well-bound and clearly presented book which continues the standard set by the IARC Scientific Publications series. Divided into 4 main sections (namely Chemistry and Formation, Analysis, Occurrence and Experimental Pathology) it is a book for reference and for the specialist concerned with the problems of the presence of nitroso-compounds in the environment. The first section is by far the largest, comprising more than one third of the volume. It contains many examples of how nitrosocompounds may be formed e.g. by treatment of corn with nitrous acid or endogenously, through inhalation of $\mathrm{NO}_{2}$, from food products and from some drugs under simulated gastric conditions. The protective effects of the vitamins, ascorbic acid and DL- $\alpha$ tocopherol, are also considered. The much shorter section on Analysis includes a comparative analysis of waste waters for the presence of nitrosamines, using a variety of detectors, as well as accounts of recent developments in the detection of volatile nitrosamines in foodstuffs.

Under the heading of Occurrence, the 
presence of nitroso-compounds is given for human and animal foodstuffs, in tobacco smoke, in the laboratory and factory environments and in confined spaces (such as inside new cars). An examination of the fate of ingested nitrate and nitrite in man and a variety of other organisms suggests that the ratio of nitrate excreted in urine to the total amount ingested is influenced by the degree of reduction to nitrite within the organism, thus indicating the possible in vivo formation of nitroso compounds.

The section Experimental Pathology includes a comparison of the degree of skin penetration by nitrosamines of environmental importance, and an interesting series of experiments suggesting that very low levels of other chemical carcinogens can contribute to the carcingoenic action of a nitrosamine.

The volume ends with a very short, but useful, set of suggestions indicating priorities for future work under the 4 main topics considered.

\section{P. J. O’CONNOR}

Gloning of Human Tumour Stem Cells. Ed. S. E. Salmon (1981). New York: Alan R. Liss Inc. 367 pp.

This volume deals in depth with a topic which is of considerable interest both to basic scientists and clinicians working in the field of cancer chemotherapy. It contains 23 chapters dealing with all aspects of cloning and drug-sensitivity testing of human tumours in vitro. However one cannot avoid the feeling that a somewhat biased view is presented since 13 of the 23 chapters are co-authored by the editor S. E. Salmon, and most of the other 10 chapters are written by members of the same group. From the data presented in the various chapters it is obvious that the Arizona group has had considerable success in cloning human tumours of a variety of histological types, and in using measurements of their drug sensitivity in vitro for both retrospective and predictive studies of the sensitivity of individual tumours to therapy. However there is little indication in this volume that the methods and results are reproducible in other laboratories. Chapters on the experiences of completely independent investigators would have greatly improved its value. Nevertheless the book represents an impressive collection of data indicating that the tailoring of chemotherapy to the needs of individual patients is a feasible possibility, at least in the hands of some investigators. The extension of such studies to other laboratories should provide valuable information both on the biology and drug sensitivity of human tumours and could considerably improve treatment in the future.

M. Fox

A Short Textbook of Radiotherapy. 4th Edition. J. Walter, H. Miller and C. K. Bomford (1979). Edinburgh: Churchill Livingstone. 299 pp. $£ 11$ net.

This book has been written primarily for student radiotherapy radiographers working for the qualifying diploma of the College of Radiographers. It covers most of the syllabus for this examination in varying depth and should be used in conjunction with lecture notes and reference to other works listed in the bibliography. Ten years have elapsed since the last edition, and the authors have eliminated many outdated practices from the text.

Part $I$ is on radiation physics. The initial chapters cover radioactivity, the production and measurement of X-ray and gamma-ray beams, and their interaction with matter. The physical principles of radiation treatment planning, the use of the simulator and mould-room technique are fully explained. The chapter on the use of sealed radioactive sources includes radon seeds, but does not state that these are no longer produced in the United Kingdom. The Paterson-Parker rules are given for the distribution of sources and dose calculations for surface applicators, interstitial implantation and intracavitary therapy, and afterloading techniques are mentioned. There are 2 chapters on unsealed sources. Radiation protection procedures have their due prominence and abstracts are included of the U.K. Code of Practice. The reader is also introduced to recent developments in technology such as ultrasound, computerized transverse axial tomography, and computers for treatment planning and dose calculations.

Part II on radiotherapy and oncology starts with general chapters on "the cancer problem and pathology". There is an interesting chapter on the public-health aspects of cancer, including cancer education. The 